\title{
Gelsolin impairs barrier function in pancreatic ductal epithelial cells by actin filament depolymerization in hypertriglyceridemia-induced pancreatitis in vitro
}

\author{
HUI-YING YANG ${ }^{1}$, ZHI-HAI LIANG ${ }^{1}$, JIN-LIAN XIE ${ }^{1}$, QING WU ${ }^{2}$, \\ YING-YING QIN ${ }^{1}$, SHI-YU ZHANG ${ }^{1}$ and GUO-DU TANG ${ }^{1}$ \\ ${ }^{1}$ Department of Gastroenterology, The First Affiliated Hospital of Guangxi Medical University, Nanning, Guangxi 530021; \\ ${ }^{2}$ Department of Gastroenterology, The Second Affiliated Hospital of Guangxi Medical University, \\ Nanning, Guangxi 530007, P.R. China
}

Received October 16, 2021; Accepted January 25, 2022

DOI: $10.3892 /$ etm.2022.11219

\begin{abstract}
Gelsolin (GSN) is a calcium-regulated actin-binding protein that can sever actin filaments. Notably, actin dynamics affect the structure and function of epithelial barriers. The present study investigated the role of GSN in the barrier function of pancreatic ductal epithelial cells (PDECs) in hypertriglyceridemia-induced pancreatitis (HTGP). The human PDEC cell line HPDE6-C7 underwent GSN knockdown and was treated with caerulein $(\mathrm{CAE})+$ triglycerides $(\mathrm{TG})$. Intracellular calcium levels and the actin filament network were analyzed under a fluorescence microscope. The expression levels of GSN, E-cadherin, nectin-2, ZO-1 and occludin were evaluated by reverse transcription-quantitative polymerase chain reaction and western blotting. Ultrastructural changes in tight junctions were observed by transmission electron microscopy. Furthermore, the permeability of PDECs was analyzed by fluorescein isothiocyanate-dextran fluorescence. The results revealed that $\mathrm{CAE}+\mathrm{TG}$ increased intracellular calcium levels, actin filament depolymerization and GSN expression, and increased PDEC permeability by decreasing the expression levels of E-cadherin, nectin-2, ZO-1 and occludin compared with the control. Moreover, changes in these markers, with the exception of intracellular calcium levels, were reversed by silencing GSN. In conclusion, GSN may disrupt barrier function in PDECs by causing actin filament depolymerization in HTGP in vitro.
\end{abstract}

Correspondence to: Professor Guo-Du Tang, Department of Gastroenterology, The First Affiliated Hospital of Guangxi Medical University, 6 Shuangyong Road, Nanning, Guangxi 530021, P.R. China

E-mail: tangguodu@stu.gxmu.edu.cn

Key words: calcium, gelsolin, actin filament, barrier function, pancreatic ductal epithelial cells, hypertriglyceridemia-induced pancreatitis

\section{Introduction}

Severe hypertriglyceridemia (HTG) is the third major cause of acute pancreatitis (AP) after alcohol abuse and cholelithiasis worldwide (1). The severity and complication rates of HTG-induced pancreatitis (HTGP) are higher than those of AP that originate from other causes (2). The mechanism by which high triglyceride (TG) levels trigger AP is unknown (3), but may be due to the toxicity of free fatty acids (FFAs) to the pancreas. High concentrations of FFAs are generated from TG hydrolysis by lipases in pancreatic tissues and may trigger the self-digestion of the pancreas by damaging pancreatic acinar cells (PACs) and vascular endothelial cells, activating trypsinogen and protein kinase $\mathrm{C}$, and releasing intracellular calcium $(3,4)$.

Pancreatic ductal epithelial cells (PDECs) are considered the most important component of the pancreatic duct mucosal barrier, and serve a critical role in preventing the reflux of bile and pancreatic enzymes (5). Previous studies have reported that barrier disfunction in PDECs is a major contributor to the occurrence of pancreatitis (6-9). Our previous study revealed that the synthetic cholecystokinin analogue caerulein (CAE) induced AP in the human pancreatic ductal epithelial cell line HPDE6-C7 (10). In addition, high-fat diet-derived FFAs can impair intestinal epithelial cell permeability (11-13); nonetheless, the effect of HTG on PDECs in AP is incompletely understood.

The cytoskeleton is a complex and dynamic system composed primarily of actin filaments, which is essential for key cellular functions, including adhesion, spreading, migration and interaction with the environment (14). Previous studies have reported that the maintenance of the endothelial barrier function and cell-cell junctions is critically dependent on actin filament dynamics (15-18), which are regulated by actin-binding proteins (ABPs) (19). Moreover, FFAs can modulate actin reorganization and have toxic effects on PACs by increasing cytosolic calcium concentrations (20-22), potentially leading to HTGP. However, to the best of our knowledge, the underlying mechanism of actin dynamics in PDECs in HTGP has not been explored. 
Gelsolin (GSN) is a calcium-regulated ABP that controls actin dynamics by nucleating, capping and severing actin filaments, and participates in cell morphology, motility, metabolism, apoptosis and phagocytosis (23). Moreover, GSN has been reported to be involved in cell-cell junctions and can regulate cell adhesion strength by remodeling actin $(24,25)$. Our unpublished data revealed that GSN is expressed in PDECs (HPDE6-C7) (Yang et al, unpublished data); however, the role of GSN in PDECs in HTGP is not fully understood.

The present study hypothesized that GSN may impair barrier function in PDECs by regulating actin filaments in HTGP. To test this hypothesis, GSN gene expression was knocked down and HTGP was induced using CAE (13) and TG in HPDE6-C7 cells, in order to assess the effect of GSN-regulated actin filaments on barrier function in PDECs in HTGP in vitro.

\section{Materials and methods}

Cell culture. The human PDEC line HPDE6-C7 (Guangzhou Jenniobio Biotechnology) was cultured in DMEM (Gibco; Thermo Fisher Scientific, Inc.) supplemented with $10 \%$ fetal bovine serum (Shanghai Shuangru Biotechnology Co., Ltd.), 1\% L-glutamine (Beijing Solarbio Science \& Technology Co., Ltd.) and $1 \%$ penicillin-streptomycin mixture (Beijing Solarbio Science \& Technology Co., Ltd.) at $37^{\circ} \mathrm{C}$ in a humidified atmosphere containing $5 \% \mathrm{CO}_{2}$. Cells were grown on coverslips $(24 \times 24 \mathrm{~mm})$ in 6 -well plates or $25-\mathrm{cm}^{2}$ flasks.

RNA interference-mediated GSN gene silencing. Three pairs of short hairpin RNA (shRNA) sequences were designed by BLOCK-iT ${ }^{\mathrm{TM}}$ RNAi Designer (Thermo Fisher Scientific Inc.) according to GSN coding DNA sequences and were synthesized by Sangon Biotech Co., Ltd. (Table I). The third generation lentiviral packaging system was used to inhibit GSN expression analysis. The shRNAs were cloned into the pcDNA6.2-GW/EmGFP-miR plasmid vector (Shanghai R\&S Biotechnology Co., Ltd.). The cloned DNA fragments were amplified by PCR and subcloned into the pLenti6.3/V5-DEST vector (Shanghai R\&S Biotechnology Co., Ltd.). A total of $60 \mu \mathrm{g}$ lentiviral plasmids and ViraPower ${ }^{\mathrm{TM}}$ lentiviral packaging mix (Invitrogen; Thermo Fisher Scientific Inc.), mixed with POLOdeliverer ${ }^{\text {TM }} 3000$ Transfection Reagent (Shanghai R\&S Biotechnology Co., Ltd.), were co-transfected into 293T cells (Shanghai R\&S Biotechnology Co., Ltd.) in a 1:1 ratio. The transfected cells were incubated at $37^{\circ} \mathrm{C}$ for 48 and $72 \mathrm{~h}$, respectively, and the lentivirus supernatant was collected and concentrated. HPDE6-C7 cells were infected with lentivirus for $72 \mathrm{~h}$, with a multiplicity of infection of 8 . The lentivirus-infected cells derived from the third pairs of shRNAs were selected for subsequent experiments by reverse transcription-quantitative polymerase chain reaction (RT-qPCR) screening and validation. The stable cell lines were created using $1.0 \mu \mathrm{g} / \mathrm{ml}$ blasticidin for selection and $0.5 \mu \mathrm{g} / \mathrm{ml}$ for maintenance. The lentivirus obtained from empty pLenti6.3/V5-DEST was used as an empty vector and HPDE6-C7 cells transduced with an empty vector were used as the negative control (NC).

Cell treatment and experimental grouping. Cells with or without GSN silencing were treated with $100 \mathrm{nM}$ CAE (MilliporeSigma), 2.5 mM TG (cat. no. T9420; Beijing
Solarbio Science \& Technology Co., Ltd.), or CAE + TG for $24 \mathrm{~h}$ at $37^{\circ} \mathrm{C}$. CAE was resuspended in phosphate-buffered saline (PBS), and TG was resuspended in PBS containing $0.1 \mathrm{mg} / \mathrm{ml}$ bovine serum albumin (cat. no. A8020; Beijing Solarbio Science \& Technology Co., Ltd.). AP was induced in vitro using $\mathrm{CAE}$, the effects of $\mathrm{HTG}$ on PDECs were assessed using TG, and HTGP was induced by CAE + TG. Cells were divided into 12 groups, as follows: Blank control (BC) group, cells without lentiviral transduction; CAE-treated BC group (CAE group); TG-treated BC group (TG group); $\mathrm{CAE}+\mathrm{TG}$-treated BC group (CAE + TG group); NC group, cells transduced with an empty vector; CAE-treated NC group (NC + CAE group); TG-treated NC group ( NC + TG group); $\mathrm{CAE}+\mathrm{TG}$-treated NC group (NC + CAE + TG group); knockdown (KD) group, cells with GSN KD; CAE-treated KD group (KD + CAE group); TG-treated KD group (KD + TG group); and $\mathrm{CAE}+\mathrm{TG}$-treated $\mathrm{KD}$ group (KD + CAE + TG group).

Inverted biological microscopy analysis of cell morphology. Cells at a density of $4 \times 10^{5}$ cells/well were seeded into a six-well plate and cultured at $37^{\circ} \mathrm{C}$ in a humidified atmosphere containing $5 \% \mathrm{CO}_{2}$ until they reached $75-80 \%$ confluence. Then the cells were treated with purified trilaurin (TG; cat. no. T9420; Beijing Solarbio Science \& Technology Co., Ltd.) at concentrations of $0.5,1.0,2.5,5$ and $10 \mathrm{mM}$ for $24 \mathrm{~h}$ at $37^{\circ} \mathrm{C}$. Cell morphology was analyzed by an inverted biological microscopy (CKX53; Olympus Corporation) at x100 magnification.

Cell Counting Kit-8 (CCK-8) assay. Cell viability was determined using CCK-8 (cat. no. CA1210; Beijing Solarbio Science \& Technology Co., Ltd.) according to the manufacturer's protocols. HPDE6-C7 cells at a density of $1 \times 10^{4}$ cells/well were seeded into a 96-well plate with $100 \mu 1$ growth medium/well and cultured for $12 \mathrm{~h}$. Subsequently, the cells were treated with TG $(10 \mu \mathrm{l} /$ well $)$ at concentrations of $0.5,1.0,2.5,5.0$ and $10 \mathrm{mM}$ for $24 \mathrm{~h}$ at $37^{\circ} \mathrm{C}$ and $10 \mu \mathrm{lCCK}-8$ reagent was added to each well for $1 \mathrm{~h}$ at $37^{\circ} \mathrm{C}$. The absorbance was measured at $450 \mathrm{~nm}$ using a microplate reader (Varioskan LUX; Thermo Fisher Scientific Inc.).

DAPI staining. The cells were seeded into a 6-well plate at a density of $1 \times 10^{5}$ cells/well and cultured for $24 \mathrm{~h}$, then treated with $100 \mathrm{nMCAE}$ for $6,12,24$ and $48 \mathrm{~h}$ or TG at concentrations of $0.5,1.0,2.5,5.0$ and $10 \mathrm{mM}$ for $24 \mathrm{~h}$ at $37^{\circ} \mathrm{C}$. Subsequently, the cells were fixed in $4 \%$ paraformaldehyde (cat. no. P1110; Beijing Solarbio Science \& Technology Co., Ltd.) for $30 \mathrm{~min}$ and stained with $100 \mu \mathrm{l}$ DAPI solution $(10 \mu \mathrm{g} / \mathrm{ml}$; cat. no. C0065; Beijing Solarbio Science \& Technology Co., Ltd.) in the dark for $5 \mathrm{~min}$ (all steps at room temperature). The cell nuclei were imaged at 460-495 nm under a fluorescence microscope. The cells stained light blue represented the DAPI positively stained cells.

Measurement of intracellular calcium. HPDE6-C7 cells were grown on $60-\mathrm{mm}$ culture dishes until they reached $60-65 \%$ confluence. The cells were then washed three times with Hanks' balanced salt solution without calcium, magnesium and phenol red (cat. no. H1046; Beijing Solarbio Science \& Technology Co., Ltd.) and were stained with Calcium Crimson $(5 \mu \mathrm{M}$; 
Table I. Short hairpin RNA sequences used for GSN silencing.

\begin{tabular}{ll} 
Name & \multicolumn{1}{c}{ Oligonucleotide sequence $\left(5^{\prime}-3^{\prime}\right)$} \\
\hline GSN-1 & F: TGCTGAAGAAGTCTCCATAAAGGTTGGTTTTGGCCACTGACTGACCAACCTTTGGAGACTTCTT \\
& R: CCTGAAGAAGTCTCCAAAGGTTGGTCAGTCAGTGGCCAAAACCAACCTTTATGGAGACTTCTTC \\
GSN-2 & F: TGCTGAGAACTGTCCATATGTGGCAGGTTTTGGCCACTGACTGACCTGCCACATGGACAGTTCT \\
& R: CCTGAGAACTGTCCATGTGGCAGGTCAGTCAGTGGCCAAAACCTGCCACATATGGACAGTTCTC \\
GSN-3 & F: TGCTGTACAGAATGATGTAGCTGTCGGTTTTGGCCACTGACTGACCGACAGCTATCATTCTGTA \\
& R: CCTGTACAGAATGATAGCTGTCGGTCAGTCAGTGGCCAAAACCGACAGCTACATCATTCTGTAC
\end{tabular}

F, forward; GSN, gelsolin; R, reverse. Underlined text indicates effective RNA interference target sequences.

Table II. Target gene primers used in reverse transcription-quantitative polymerase chain reaction.

\begin{tabular}{lll}
\hline Gene & \multicolumn{1}{c}{ Forward primer (5'-3') } & Reverse primer (5'-3') \\
\hline GAPDH & ACATCGCTCAGACACCA & GTAGTTGAGGTCAATGAAGGG \\
GSN & AGCTGGCCAAGCTCTACAAG & TGTTTGCCTGCTTGCCTTTC \\
E-cadherin & AGGATGACACCCGGGACAAC & TGCAGCTGGCTCAAGTCAAAG \\
Nectin-2 & ACCTGCAAAGTGGAGCATGAGA & CCGGAGATGGACACTTCAGGA \\
ZO-1 & ATAAAGTGCTGGCTTGGTCTGTTTG & GCACTGCCCACCCATCTGTA \\
Occludin & AGTGCCACTTTGGCATTATGAGA & CTTGTGGCAGCAATTGGAAAC \\
\hline
\end{tabular}

GSN, gelsolin.

$1.0 \mathrm{ml} / \mathrm{dish}$; cat. no. C3018; Invitrogen; Thermo Fisher Scientific, Inc.) at $37^{\circ} \mathrm{C}$ for $30 \mathrm{~min}$ in the dark. Subsequently, cells were washed with D-HBSS three times and stained with Hoechst $33258(30 \mu \mathrm{g} / \mathrm{ml} ; 1.0 \mathrm{ml} /$ dish; cat. no. H3569; Invitrogen; Thermo Fisher Scientific, Inc.) at $37^{\circ} \mathrm{C}$ for $20 \mathrm{~min}$ in the dark. Fluorescence in the cytosol and nucleus were measured at 530-550 and 460-495 nm, respectively, under an inverted fluorescence microscope (IX71; Olympus Corporation). Images were analyzed using ImageJ software (version 1.8.0; National Institutes of Health).

Transmission electron microscopy (TEM). Control and GSN-silenced HPDE6-C7 cells were cultured in $25-\mathrm{cm}^{2}$ flasks until they reached $70-80 \%$ confluence. Cells were harvested using a cell scraper, centrifuged at $1,500 \mathrm{x} g$ for $10 \mathrm{~min}$ at room temperature, fixed in $3 \%$ glutaraldehyde for $2.5 \mathrm{~h}$ at $4^{\circ} \mathrm{C}$ and washed three times with PBS (10 mM; $10 \mathrm{~min} /$ wash). Subsequently, the samples were fixed in $1 \%$ osmium for $2 \mathrm{~h}$ at $4^{\circ} \mathrm{C}$, washed three times with PBS (10 min/wash), dehydrated through a graded ethanol series, embedded in epoxy resin at $37^{\circ} \mathrm{C}$ overnight. Epoxy resin samples were heated at $35^{\circ} \mathrm{C}$ for $12 \mathrm{~h}, 45^{\circ} \mathrm{C}$ for $15 \mathrm{~h}$ and $60^{\circ} \mathrm{C}$ for $24 \mathrm{~h}$, successively, cut into $70-\mathrm{nm}$ sections and stained with $3 \%$ uranium acetate-lead citrate. Ultrastructural changes in tight junctions (TJs) were analyzed using TEM (Hitachi, Ltd.).

Western blotting. Proteins were lysed from HPDE6-C7 cells using RIPA buffer (Beijing Solarbio Science \& Technology Co., Ltd.) containing $1 \%$ phenylmethyl sulfonyl fluoride (Beijing Solarbio Science \& Technology Co., Ltd.) on ice for
$30 \mathrm{~min}$, and were centrifuged at $12,000 \mathrm{x}$ g for $20 \mathrm{~min}$ at $4^{\circ} \mathrm{C}$. Protein concentration was determined using a bicinchoninic acid protein assay kit (cat. no. P0012; Beyotime Institute of Biotechnology). Proteins samples (50 $\mu \mathrm{g} /$ well) were separated by $10 \%$ sodium dodecyl sulfate-polyacrylamide gel electrophoresis and electrotransferred to polyvinylidene fluoride membranes. Membranes were then blocked with 5\% non-fat milk for $1 \mathrm{~h}$ at room temperature and incubated with primary antibodies against GSN (1:1,000; cat. no. ab74420; Abcam), ZO-1 (1:1,000; cat. no. 21173-1-AP; Wuhan Sanying Biotechnology), nectin-2 (1:1,000; cat. no. ab135246; Abcam), E-cadherin (1:1,000; cat. no. 20874-1-AP; Wuhan Sanying Biotechnology), occludin (1:1,000; cat. no. 27260-1-AP; Wuhan Sanying Biotechnology) and GAPDH (1:10,000; cat. no. ab181602; Abcam) overnight at $4^{\circ} \mathrm{C}$. Subsequently, membranes were incubated with DyLight 680-conjugated secondary anti-rabbit antibody (1:10,000; cat. no. 5366; Cell Signaling Technology, Inc.) for $1 \mathrm{~h}$ at room temperature in the dark. Immunoreactive bands were imaged using the Odyssey infrared imaging system (LI-COR Biosciences) and fluorescence intensity was semi-quantified using ImageJ software (version 1.8.0; National Institutes of Health).

$R T$ - $q P C R$. Total RNA was isolated from HPDE6-C7 cells using RNAiso Plus (cat. no. 9108; Takara Bio, Inc.) and converted to cDNA by RT using PrimeScript RT reagent kit with gDNA Eraser (cat. no. RR047B; Takara Bio, Inc.) according to manufacturer's protocol. qPCR was performed using TB Green Premix Ex Taq II (cat. no. RR820B; Takara Bio, Inc.) and corresponding primers (Table II). The thermocycling 
conditions were as follows: Pre-denaturation at $95^{\circ} \mathrm{C}$ for $30 \mathrm{sec}$, followed by 40 cycles of denaturation at $95^{\circ} \mathrm{C}$ for $5 \mathrm{sec}$, annealing and extension at $60^{\circ} \mathrm{C}$ for $34 \mathrm{sec}$. GAPDH was used as internal reference gene and the relative expression levels of target genes were measured using the $2^{-\Delta \Delta \mathrm{Cq}}$ method (26).

Immunofluorescence staining of actin filaments. Cells were plated onto coverslips $(24 \times 24 \mathrm{~mm})$ in 6-well plates until they reached $55-60 \%$ confluence. The cells were fixed in $4 \%$ paraformaldehyde for $15 \mathrm{~min}$ at room temperature, permeabilized in $0.1 \%$ Triton X-100 for $5 \mathrm{~min}$ at room temperature, blocked in PBS containing $2 \%$ bovine serum albumin for $20 \mathrm{~min}$ at room temperature and stained with tetramethyl rhodamine isothiocyanate-phalloidin (100 nM; $50 \mu \mathrm{l} /$ well; Beijing Solarbio Science \& Technology Co., Ltd.) for $30 \mathrm{~min}$ at room temperature in the dark. The samples were washed with PBS three times at 5-min intervals between each step and mounted with anti-fading medium containing DAPI (cat. no. S2100; Beijing Solarbio Science \& Technology Co., Ltd.) for $3 \mathrm{~min}$ at room temperature in the dark. Actin filaments were observed at 530-550 nm and cell nuclei were observed at 460-495 nm under an upright fluorescence microscope (BX53; Olympus Corporation).

Fluorescein isothiocyanate (FITC)-dextran fluorescence. HPDE6-C7 cell suspensions $\left(2 \times 10^{5}\right.$ cells/well) were cultured on microporous polycarbonate membranes (pore size, $0.4 \mu \mathrm{m}$ ) until they reached $100 \%$ confluence in the upper compartment of 12-well Transwell plates (cat. no. 3401; Corning, Inc.). The culture medium in the upper compartment was removed and adhered cells were washed with PBS twice. Subsequently, FITC-dextran (4 kDa; $0.5 \mathrm{mg} / \mathrm{ml} ; 500 \mu \mathrm{l} /$ well; MilliporeSigma) was added to the upper compartment, and PBS ( $1 \mathrm{ml} /$ well) was added to the lower compartment. The plates were incubated in the dark for $60 \mathrm{~min}$ at $37^{\circ} \mathrm{C}$. Fluorescence in the lower compartment was measured using a microplate reader (excitation, $495 \mathrm{~nm}$; emission, $520 \mathrm{~nm}$; Thermo Fisher Scientific, Inc.) and quantified using a calibration curve of FITC-dextran.

Statistical analysis. Data are presented as the mean \pm standard deviation and the experiments were repeated at least three times. Statistical analysis was performed by one-way analysis of variance and Tukey's post hoc test using SPSS Statistics version 22.0 (IBM Corp.), GraphPad Prism version 6.0 (GraphPad Software, Inc.) and ImageJ (version 1.8.0; National Institutes of Health). $\mathrm{P}<0.05$ was considered to indicate a statistically significant difference.

\section{Results}

Effect of TG on cell viability and cell-cell junctions of PDECs. Cell viability and cell apoptosis of PDECs treated with TG $(0.5,1.0,2.5,5.0$ and $10 \mathrm{mM})$ for $24 \mathrm{~h}$ were assessed by light microscope, CCK-8 assay and DAPI staining. The optimal TG intervention concentration was selected and its effects on cell-cell junctions was analyzed by TEM. The results showed that high levels of TG ( $\geq 2.5 \mathrm{mM})$ significantly inhibited cell viability (Fig. S1A and B) and increased cell apoptosis of PDECs (Fig. S1C). Therefore, $2.5 \mathrm{mM}$ of TG was selected for subsequent experiments. Moreover, the analysis of cell-cell junctions showed that CAE and CAE + TG disrupted cell-cell junctions, particularly TJs, compared with the control. In addition, the disruptions were more pronounced with CAE + TG, but TG had no effect on cell-cell junctions compared with the control (Fig. S2).

Effect of intracellular calcium levels on GSN expression in HPDE6-C7 cells treated with CAE and TG. Intracellular calcium was semi-quantified by fluorescence microscopy, and changes in GSN gene and protein expression levels in HPDE6-C7 cells treated with CAE and TG were analyzed by RT-qPCR and western blotting. The results revealed that $\mathrm{CAE}$ and $\mathrm{CAE}+\mathrm{TG}$, as treatments of the $\mathrm{BC}$ and NC groups, increased intracellular calcium levels (Fig. 1A and B) and the mRNA and protein expression levels of GSN relative to baseline (Fig. 1C-E), and the increase was more pronounced with $\mathrm{CAE}+\mathrm{TG}$. Notably, as treatment of the BC and NC groups, TG had no detectable effect on intracellular calcium levels (Fig. 1A and B) and GSN expression (Fig. 1C-E). Moreover, GSN silencing in the KD + CAE and KD + CAE + TG groups did not affect intracellular calcium levels compared with the $\mathrm{NC}+\mathrm{CAE}$ and $\mathrm{NC}+\mathrm{CAE}+\mathrm{TG}$ groups (Fig. 1A and B), and lentiviral-mediated RNA interference in the KD group significantly reduced the mRNA and protein expression of GSN in PDECs compared with the NC group (Fig. 1C-E).

Effect of GSN on actin filament dynamics in HPDE6-C7 cells treated with $C A E$ and TG. Changes in actin filament dynamics by GSN silencing were analyzed by immunofluorescence. The results demonstrated that $\mathrm{CAE}$ and $\mathrm{CAE}+\mathrm{TG}$, as treatments of the $\mathrm{BC}$ and $\mathrm{NC}$ groups, disrupted the actin filament network, and the effect was stronger with CAE + TG, whereas GSN silencing reduced this effect in the comparisons of the $\mathrm{NC}+\mathrm{CAE}$ and $\mathrm{KD}+\mathrm{CAE}$ groups or the $\mathrm{NC}+\mathrm{CAE}+\mathrm{TG}$ and $\mathrm{KD}+\mathrm{CAE}+\mathrm{TG}$ groups. Notably, TG, as treatment of the BC and NC groups, did not cause actin depolymerization (Fig. 2).

Effect of GSN on the major components of cell-cell junctions in HPDE6-C7 cells treated with CAE and TG. The expression levels of major components of cell-cell junctions, including E-cadherin, nectin-2, ZO-1 and occludin, were analyzed by RT-qPCR and western blotting. The results revealed that CAE and $\mathrm{CAE}+\mathrm{TG}$, as treatments of the $\mathrm{BC}$ and $\mathrm{NC}$ groups, reduced the mRNA (Fig. 3A-D) and protein (Fig. 3E-I) expression levels of these markers relative to baseline. Conversely, GSN silencing reduced the effects of CAE and CAE + TG treatment in the comparisons of the $\mathrm{NC}+\mathrm{CAE}$ and $\mathrm{KD}+\mathrm{CAE}$ groups or the $\mathrm{NC}+\mathrm{CAE}+\mathrm{TG}$ and $\mathrm{KD}+\mathrm{CAE}+\mathrm{TG}$ groups (Fig. 3).

Effect of GSN on TJ ultrastructure and permeability of HPDE6-C7 cells treated with CAE and TG. The ultrastructure of TJs in HPDE6-C7 cells was evaluated by TEM. The present study revealed that treatment with CAE and $\mathrm{CAE}+\mathrm{TG}$, as treatments of the BC and NC groups, decreased the number of TJ strands and disrupted the organization of cell-cell junctions; some cells in the CAE + TG and $\mathrm{NC}+\mathrm{CAE}+\mathrm{TG}$ groups had no TJs. GSN silencing mitigated the effects of CAE and CAE + TG treatment in the comparisons of the $\mathrm{NC}+\mathrm{CAE}$ and $\mathrm{KD}+\mathrm{CAE}$ groups 
A

A

CAE $\quad$ TG

TG CAE+TG
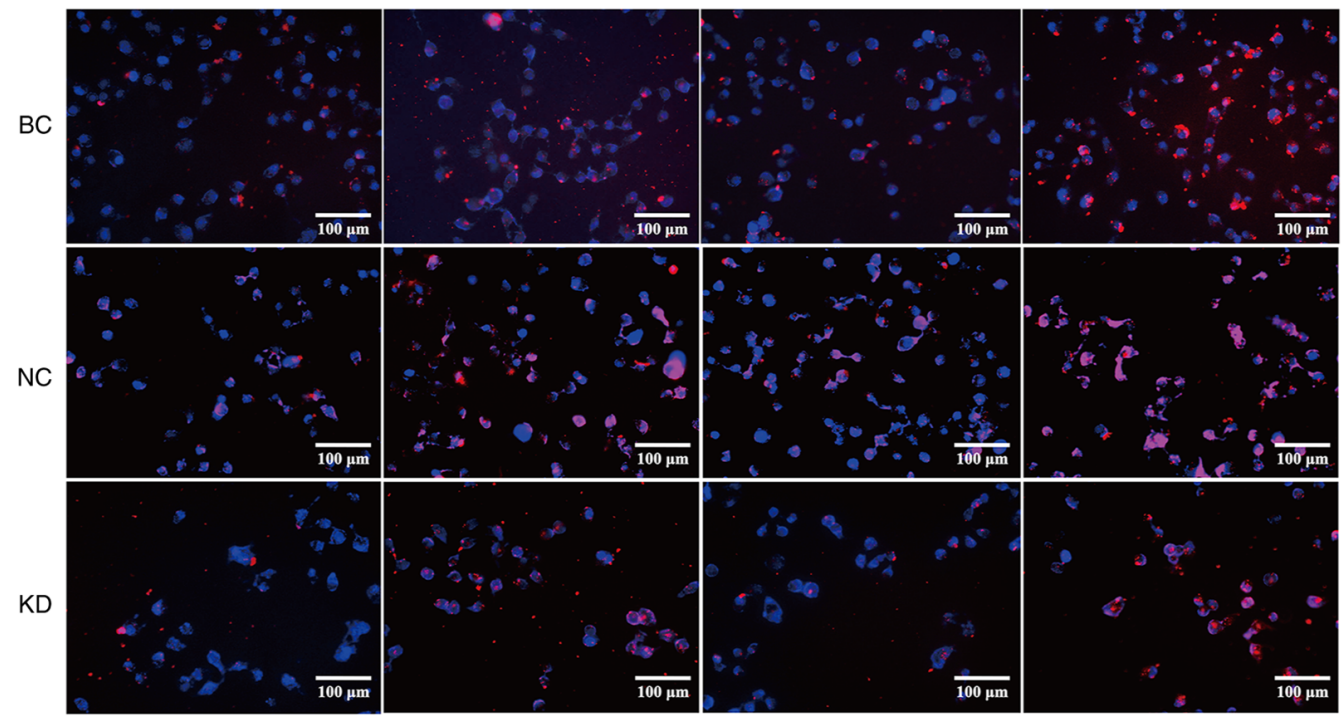

$\mathrm{B}$

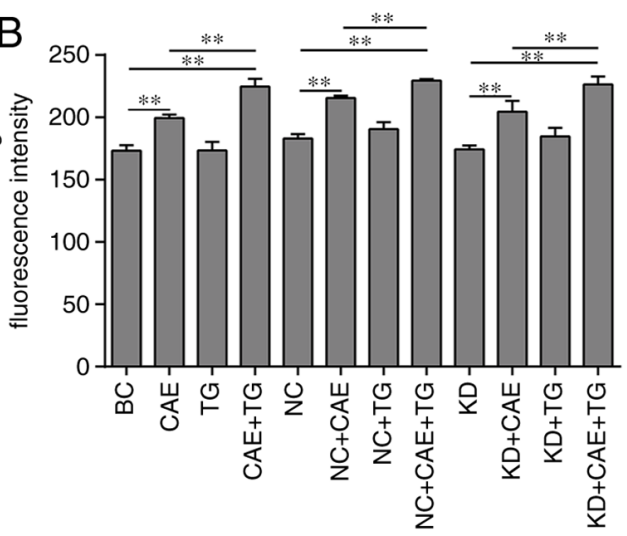

C

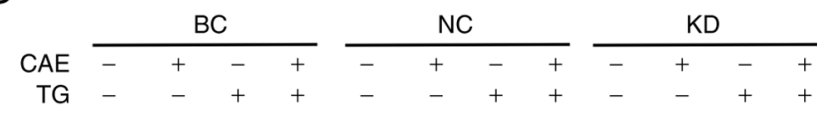

Gelsolin

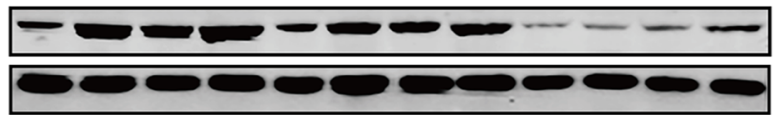

GAPDH
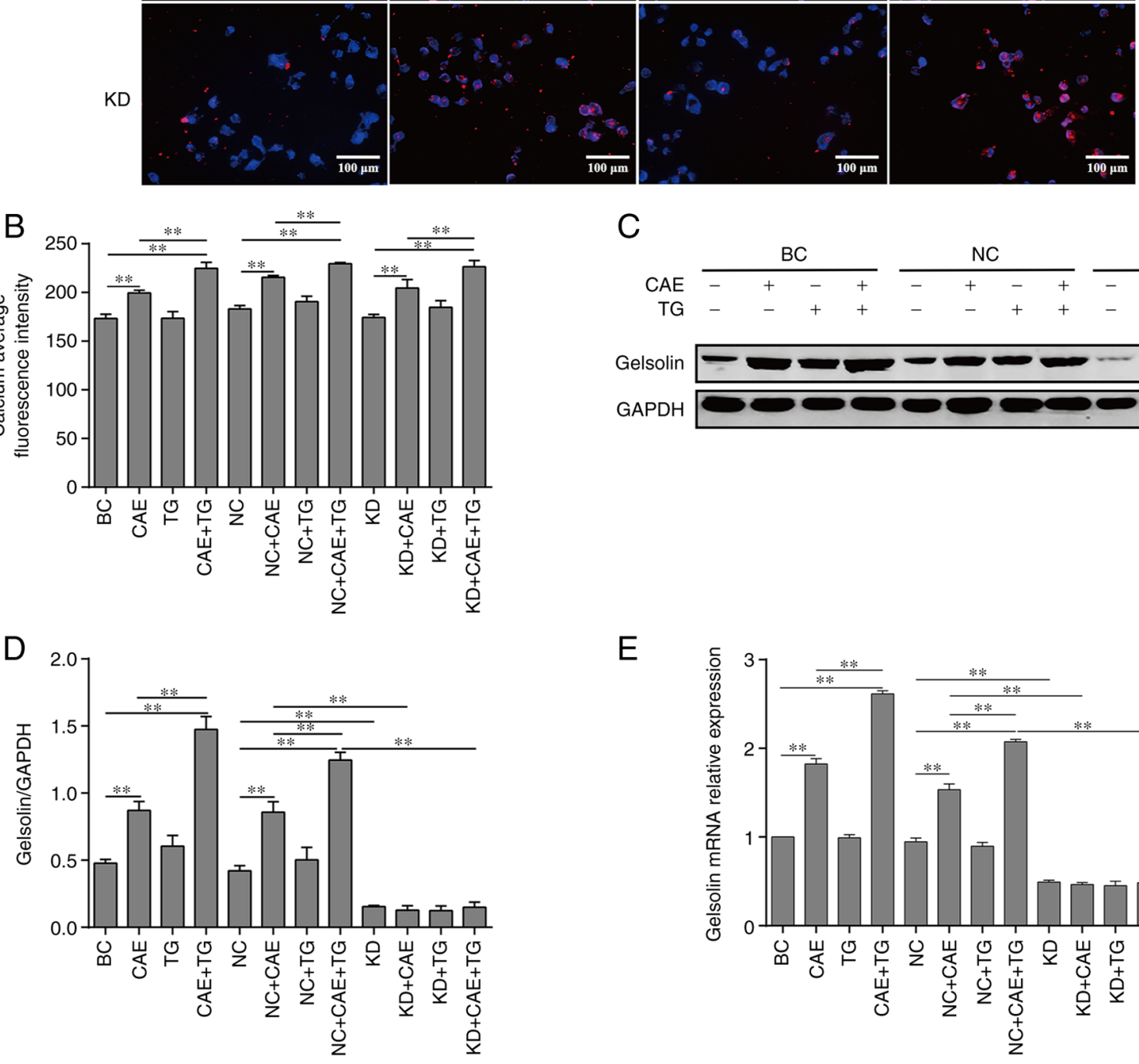

$E$

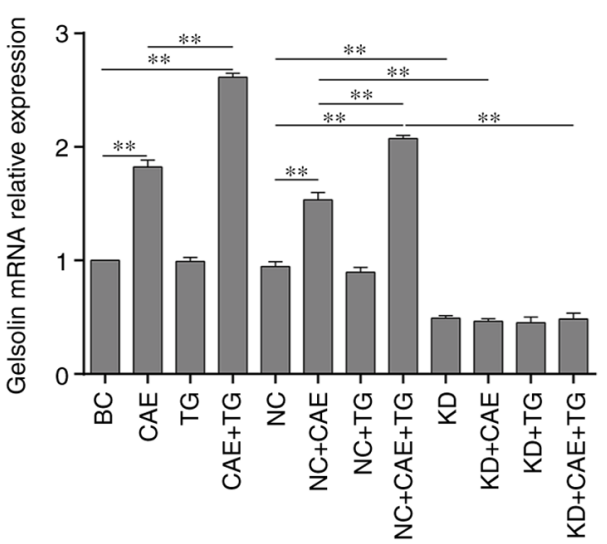

Figure 1. Effect of intracellular calcium levels on GSN expression in HPDE6-C7 cells treated with CAE and TG. (A) Changes in intracellular calcium levels based on fluorescence imaging (x200 magnification) and (B) semi-quantitative analysis. (C) Protein expression levels of GSN, as determined by western blotting and (D) semi-quantitative analysis. (E) Relative mRNA expression levels of GSN, as determined by reverse transcription-quantitative polymerase chain reaction. The experiments were repeated at least three times. Data are presented as the mean \pm standard deviation. ${ }^{* *} \mathrm{P}<0.01$. $\mathrm{BC}$, blank control; $\mathrm{CAE}$, caerulein; GSN, gelsolin; KD, knockdown; NC, negative control; TG, triglycerides.

or the $\mathrm{NC}+\mathrm{CAE}+\mathrm{TG}$ and $\mathrm{KD}+\mathrm{CAE}+\mathrm{TG}$ groups (Fig. 4A). In addition, CAE + TG, as treatment of the BC and $\mathrm{NC}$ groups, increased cell permeability more strongly than CAE, and GSN silencing attenuated this effect in the comparisons of the $\mathrm{NC}+\mathrm{CAE}$ and $\mathrm{KD}+\mathrm{CAE}$ groups or the $\mathrm{NC}+\mathrm{CAE}+\mathrm{TG}$ and $\mathrm{KD}+\mathrm{CAE}+\mathrm{TG}$ groups, whereas TG, as treatment of the $\mathrm{BC}$ and $\mathrm{NC}$ groups, alone did not affect cell permeability (Fig. 4B).
Time-dependent effects of CAE on apoptosis, GSN protein expression and actin filament dynamics in HPDE6-C7 cells. HPDE6-C7 cells were treated with CAE for 6, 12, 24 and $48 \mathrm{~h}$. Apoptosis was evaluated using DAPI staining under a fluorescence microscope, the protein expression of GSN was semi-quantified using western blotting, and actin filaments were analyzed by immunofluorescence. The results showed that CAE increased apoptosis (Fig. S3A), GSN protein 


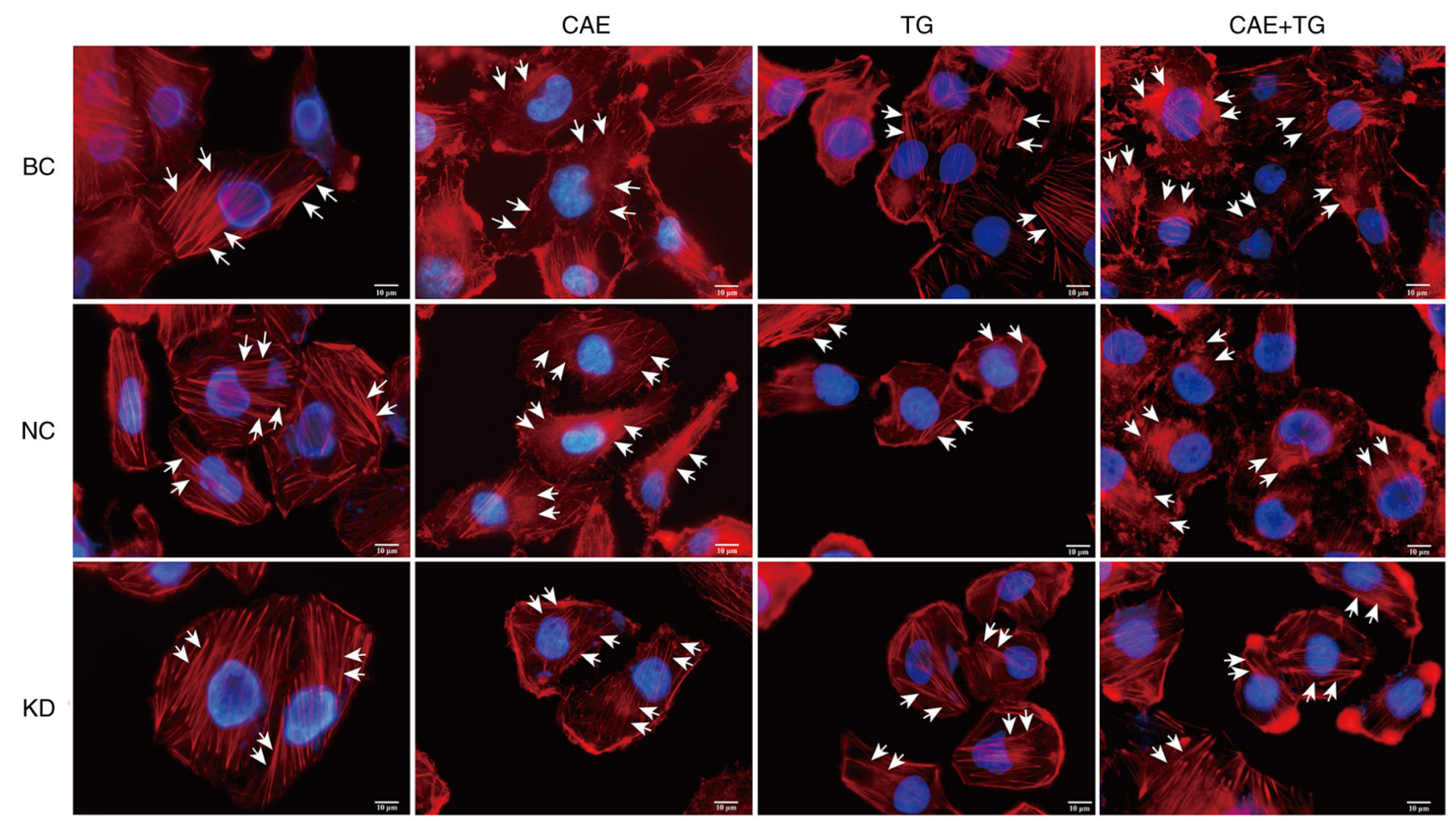

Figure 2. Effect of GSN on the dynamics of actin filaments in HPDE6-C7 cells treated with CAE and TG. The changes in actin filaments induced by silencing GSN were analyzed by tetramethyl rhodamine isothiocyanate-phalloidin immunofluorescence under an upright fluorescence microscope (x1,000 magnification, white arrows indicate actin filaments). The experiment was repeated at least three times. BC, blank control; CAE, caerulein; GSN, gelsolin; KD, knockdown; $\mathrm{NC}$, negative control; TG, triglycerides.

expression (Fig. S3B and C) and actin filament depolymerization (Fig. S3D) in a time-dependent manner in HPDE6-C7 cells compared with the control.

\section{Discussion}

Previous studies have shown that the disruption of TJs and adherens junctions, which maintain the integrity and function of the endothelial barrier in PDECs, serves an important role in the pathogenesis of $\operatorname{AP}(27,28)$. Our data showed that CAE + TG disrupted cell-cell junctions of PDECs more compared with CAE, suggesting that the impairment of barrier structure of PDECs was more pronounced in HTGP compared with in AP. In addition, actin filament dynamics are associated with the maintenance of barrier integrity in PDECs (29). Furthermore, GSN has a pivotal role in controlling the actin filament network (30). The results of the present study showed that the protein expression of GSN increased, actin filaments depolymerized and the barrier function of PDECs disrupted in an in vitro model of HTGP. Moreover, GSN silencing reduced actin filament depolymerization and barrier disruption in these cells. These data confirmed that GSN may impair barrier function in PDECs in vitro by causing the depolymerization of actin filaments in HTGP.

Calcium overload in PACs is a significant contributor to AP and also occurs in HTGP (31). GSN is a calcium-sensitive modulator of actin filament length (23); however, to the best of our knowledge, the occurrence of calcium overload and its potential association with GSN in PDECs in HTGP has not been determined. In the present study, CAE + TG increased intracellular calcium levels and GSN protein expression more strongly than CAE alone, indicating that calcium overload and GSN expression in PDECs were more pronounced in HTGP than in AP. Conversely, GSN gene silencing did not affect calcium levels, suggesting that GSN as a calcium-activated protein did not regulate calcium, which is consistent with the literature $(23,32)$.

The rearrangement of the actin network can affect the interaction between actin and ABPs (33). The ABP GSN modulates actin filament structure (30). The present study revealed that the increase in GSN expression levels and the disruption of actin dynamics were more pronounced in HTGP than in AP, whereas this disruption was prevented by knocking down GSN, indicating that GSN may depolymerize actin filaments in HTGP.

The role of GSN in cell-cell junctions in PDECs is still unclear. The present data demonstrated that the protein expression levels of GSN were increased and the cell-cell junction protein expression was decreased in the PDEC model of HTGP, whereas GSN silencing increased the expression levels of cell-cell junction proteins, indicating that GSN regulated the expression of these proteins. In addition, it has been reported that actin interacts with multiple tight junction proteins, including ZO-1 and occluding (34), and adherens junction proteins, such as nectin and E-cadherin (35). Therefore, given the regulatory effect of GSN on actin in HTGP, it was hypothesized that GSN regulated the expression of cell-cell junction proteins via actin depolymerization. However, additional studies are required to elucidate the underlying mechanisms.

Endothelial barrier function depends on the interactions of cell-cell junction proteins with the actin filament 

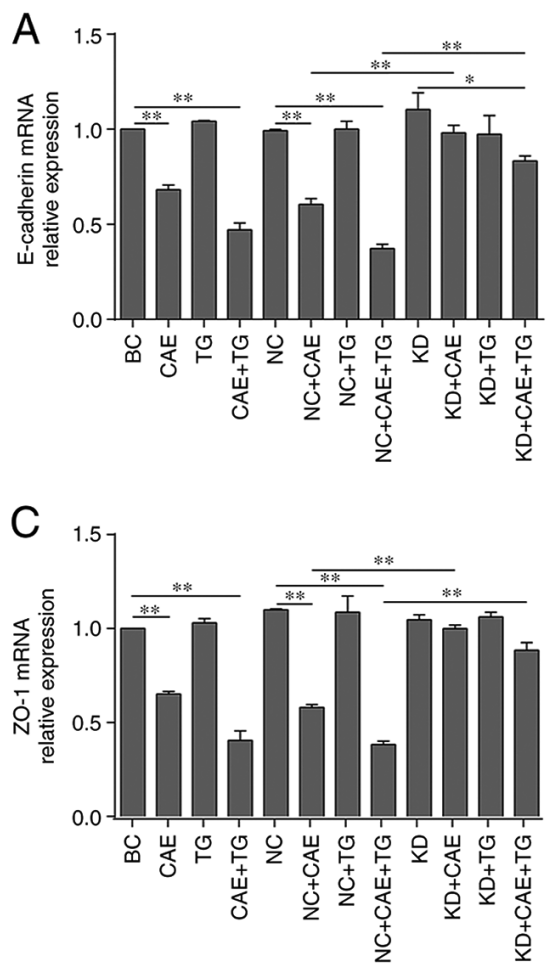

E

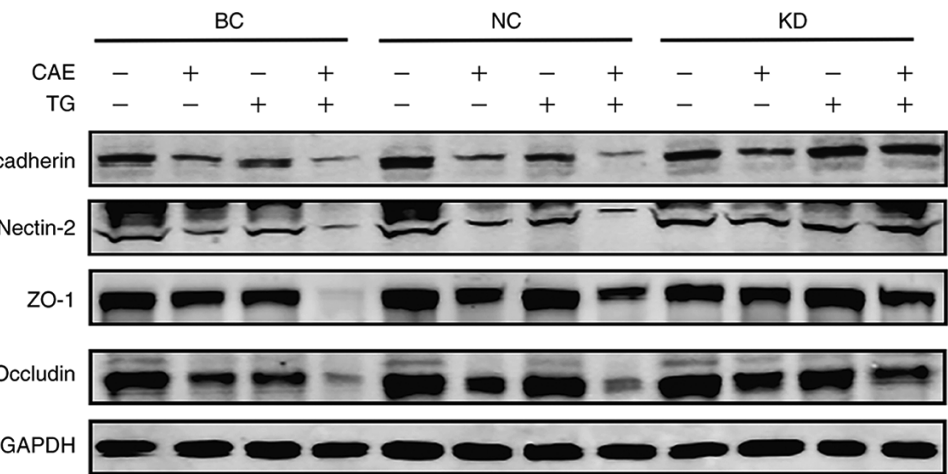

G

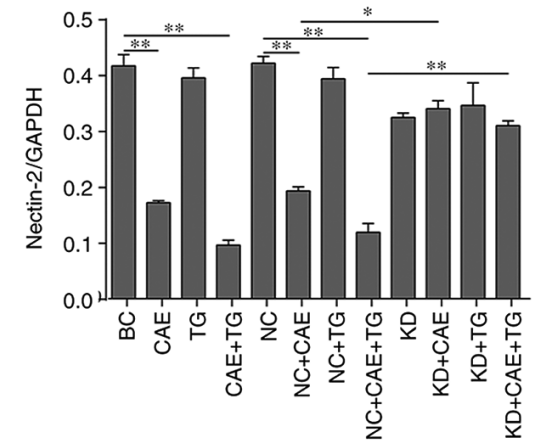

$\mathrm{H}$

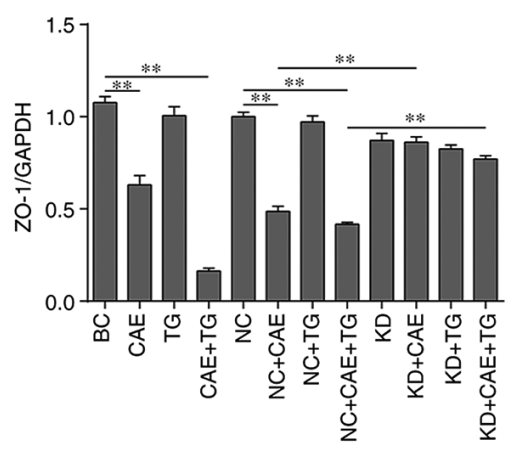

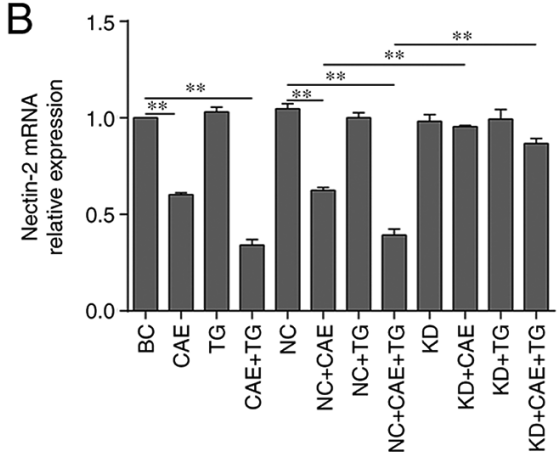

D

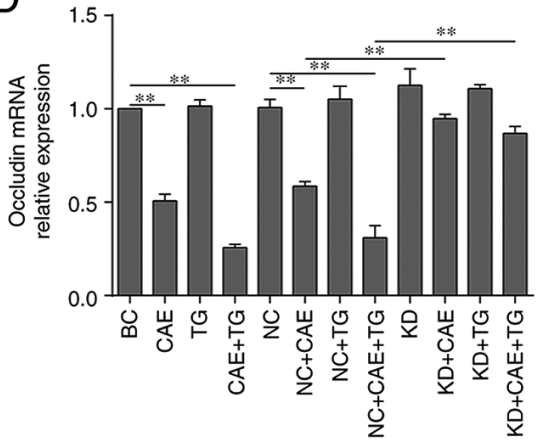

F

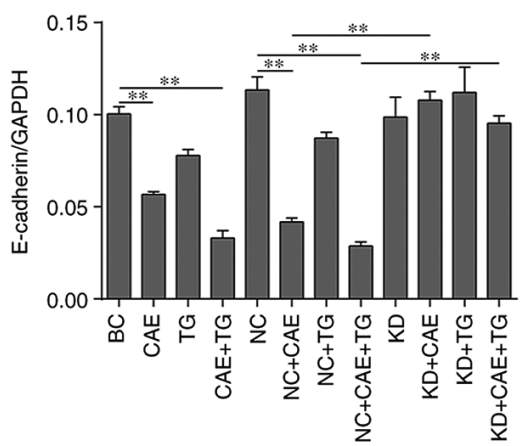

I

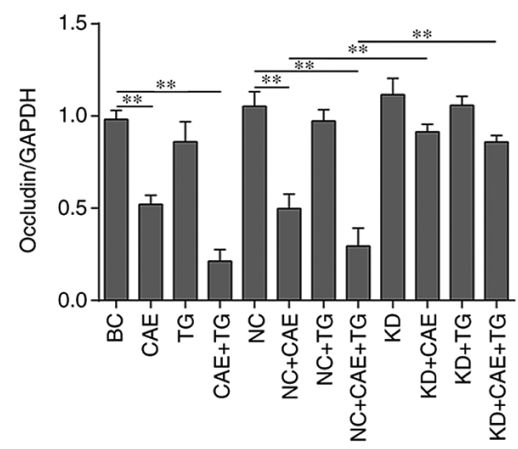

Figure 3. Effect of gelsolin on the major components of cell-cell junctions in HPDE6-C7 cells treated with CAE and TG. Relative mRNA expression levels of (A) E-cadherin, (B) nectin-2, (C) ZO-1 and (D) occludin, as determined by reverse transcription-quantitative polymerase chain reaction. (E) Western blot analysis and semi-quantification of the protein expression levels of (F) E-cadherin, (G) nectin-2, (H) ZO-1 and (I) occludin. The experiments were repeated at least three times. Data are presented as the mean \pm standard deviation. ${ }^{*} \mathrm{P}<0.05,{ }^{* *} \mathrm{P}<0.01$. BC, blank control; CAE, caerulein; KD, knockdown; $\mathrm{NC}$, negative control; TG, triglycerides.

network $(36,37)$. However, to the best of our knowledge, the role of GSN in epithelial barrier function has only been studied in ischemic lung injury (38). The present results showed that the impairment of TJs in PDECs was more severe in HTGP than in AP, and was reduced by knocking down GSN, suggesting that GSN significantly contributed to the maintenance of TJs in these cells. Moreover, the increase in the permeability of PDECs was higher in HTGP 
A

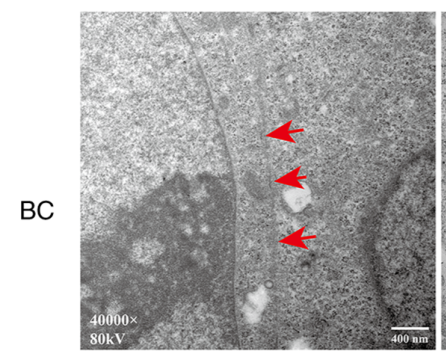

\footnotetext{
NC
}
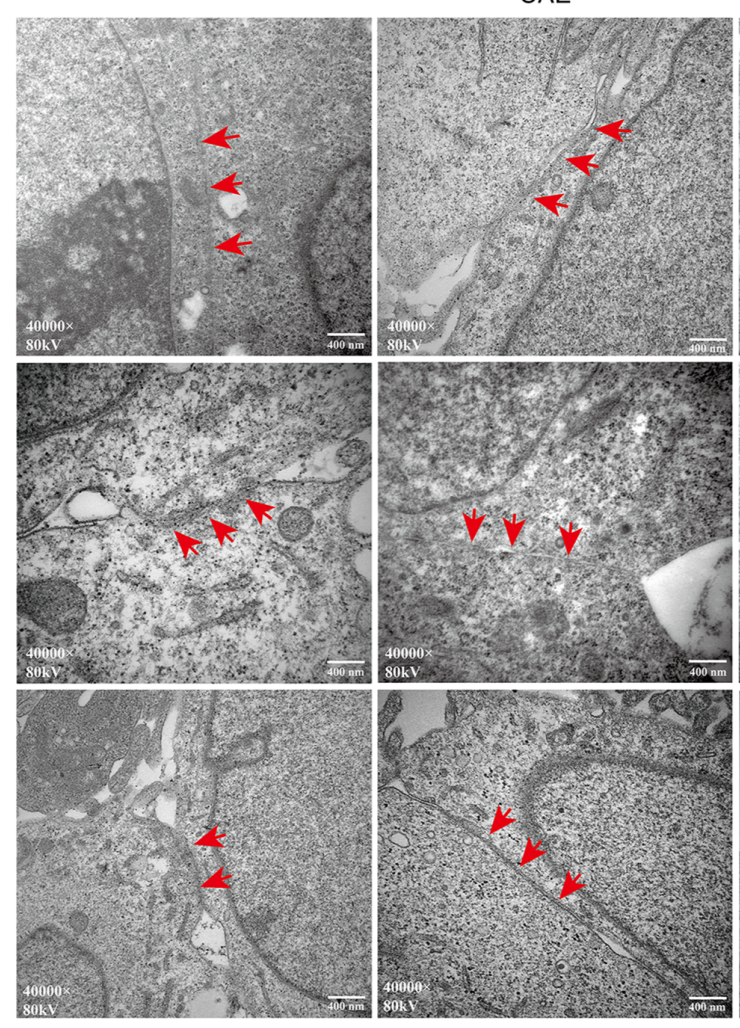

TG

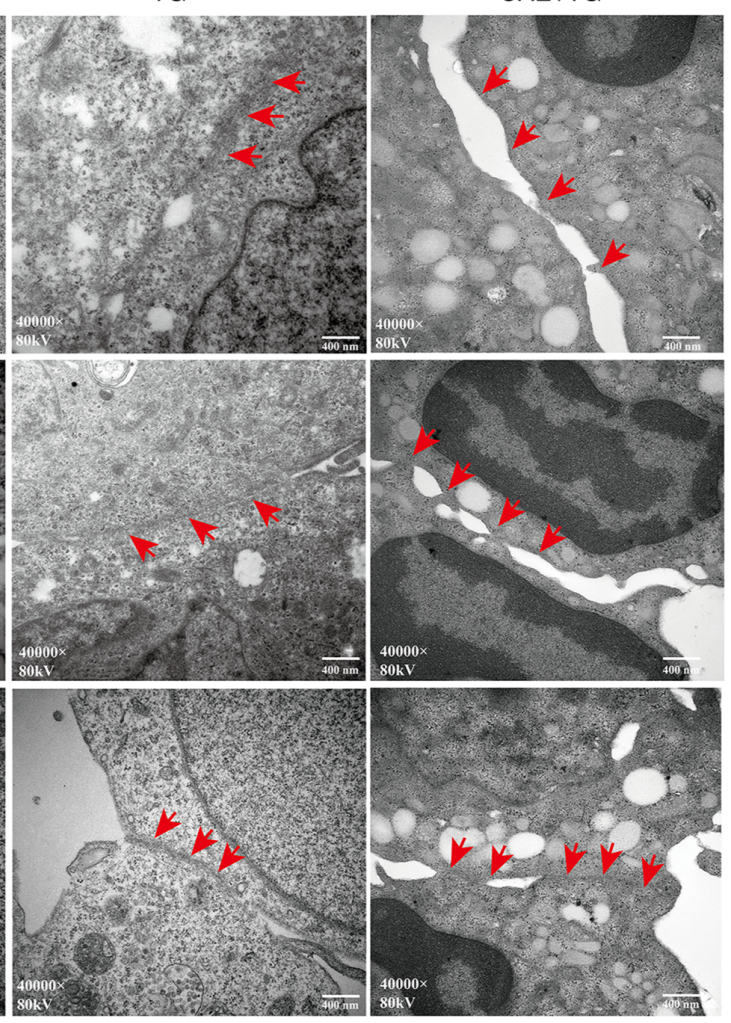

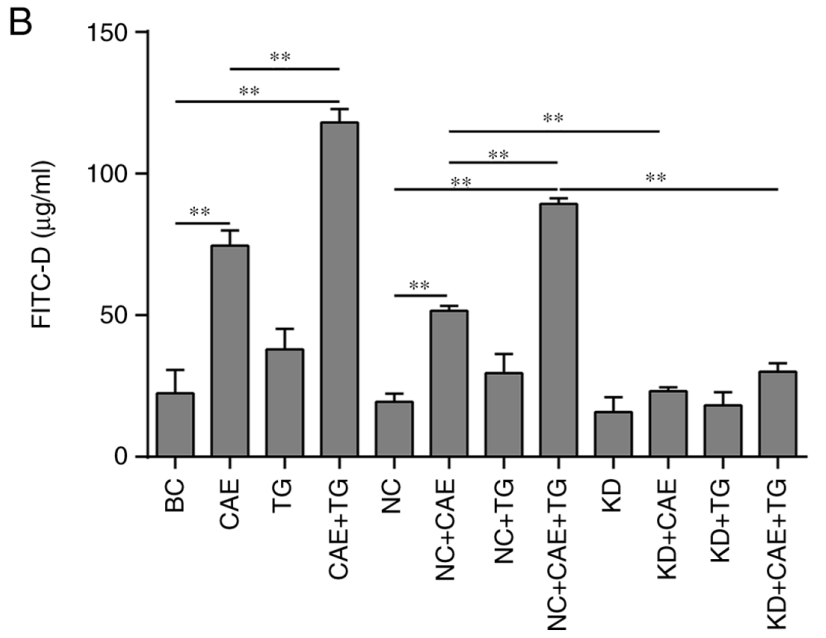

Figure 4. Effect of gelsolin on TJ ultrastructure and the permeability of HPDE6-C7 cells treated with CAE and TG. (A) Ultrastructure of TJs, as determined by transmission electron microscopy (x40,000 magnification); red arrows indicate TJs. (B) Cell permeability was analyzed by FITC-dextran fluorescence. The experiments were repeated at least three times. Data are presented as the mean \pm standard deviation. ${ }^{* *} \mathrm{P}<0.01$. BC, blank control; CAE, caerulein; KD, knockdown; NC, negative control; TG, triglycerides; TJ, tight junction.

than in AP, and this effect was reduced by GSN silencing. Therefore, considering the association between actin filaments and barrier function $(18,39)$, it was hypothesized that GSN may increase cell permeability in HTGP by depolymerizing actin.

Several limitations exist in the current study and additional work is required. Firstly, it would be beneficial to also assess the effects of GSN overexpression to further verify the regulatory mechanism. Secondly, a preliminary time-dependent study of CAE was performed and it was revealed that CAE increased apoptosis, GSN protein expression and actin filament depolymerization in a time-dependent manner in HPDE6-C7 cells (Fig. S3). Our future work will assess the time-dependent effects of TG + CAE on barrier function and actin filaments in PDECs, and the role of GSN in this mechanism.

In conclusion, the present study indicated that GSN may impair barrier function in PDECs in HTGP in vitro, potentially by causing actin depolymerization. These findings provided evidence for GSN as a novel therapeutic target for barrier disruption in PDECs in HTGP.

\section{Acknowledgements}

The authors would like to thank Mrs. Xiaomin Mo (Department of Electron Microscopy, Guangxi Medical University, 
Nanning, Guangxi, China) for her assistance in transmission electron microscopy.

\section{Funding}

The present study was supported by grants from the Guangxi Natural Science Foundation (grant no. 2018GXNSFBA281078) and the National Natural Science Foundation of China (grant no. 81970558).

\section{Availability of data and materials}

The datasets used and/or analyzed during the current study are available from the corresponding author on reasonable request.

\section{Authors' contributions}

HYY was the main contributor to the research, performing experimental work, analyzing data and writing the manuscript. GDT and ZHL conceived and designed the study. JLX and QW designed experimental procedures and performed cell culture. YYQ established an in vitro model of HTGP. SYZ performed lentiviral-mediated RNA interference and collected experimental data. HYY and GDT confirm the authenticity of all the raw data. All authors have read and approved the final manuscript.

\section{Ethics approval and consent to participate}

Not applicable.

\section{Patient consent for publication}

Not applicable.

\section{Competing interests}

The authors declare that they have no competing interests.

\section{References}

1. de Pretis N, Amodio A and Frulloni L: Hypertriglyceridemic pancreatitis: Epidemiology, pathophysiology and clinical management. United European Gastroenterol J 6: 649-655, 2018

2. Yang AL and McNabb-Baltar J: Hypertriglyceridemia and acute pancreatitis. Pancreatology 20: 795-800, 2020.

3. De Pretis N, De Marchi G and Frulloni L: Hypertriglyceridemic pancreatitis. Minerva Gastroenterol Dietol 66: 238-245, 2020.

4. Guo YY, Li HX, Zhang Y and He WH: Hypertriglyceridemiainduced acute pancreatitis: Progress on disease mechanisms and treatment modalities. Discov Med 27: 101-109, 2019.

5. Konok GP and Thompson AG: Pancreatic ductal mucosa as a protective barrier in the pathogenesis of pancreatitis. Am J Surg 117: 18-23, 1969.

6. Quilichini E, Fabre M, Dirami T, Stedman A, De Vas M, Ozguc O, Pasek RC, Cereghini S, Morillon L, Guerra C, et al: Pancreatic ductal deletion of Hnflb disrupts exocrine homeostasis, leads to pancreatitis, and facilitates tumorigenesis. Cell Mol Gastroenterol Hepatol 8: 487-511, 2019.

7. Hayashi M and Novak I: Molecular basis of potassium channels in pancreatic duct epithelial cells. Channels (Austin) 7: 432-441, 2013

8. Ishiguro H, Yamamoto A, Nakakuki M, Yi L, Ishiguro M, Yamaguchi M, Kondo S and Mochimaru Y: Physiology and pathophysiology of bicarbonate secretion by pancreatic duct epithelium. Nagoya J Med Sci 74: 1-18, 2012.
9. Maléth $\mathbf{J}$ and Hegyi P: Calcium signaling in pancreatic ductal epithelial cells: An old friend and a nasty enemy. Cell Calcium 55: 337-345, 2014.

10. Wei B, Gong Y, Yang H, Zhou J, Su Z and Liang Z: Role of tumor necrosis factor receptor-associated factor 6 in pyroptosis during acute pancreatitis. Mol Med Rep 24: 848, 2021.

11. Goldberg IJ, Cabodevilla AG, Samovski D, Cifarelli V, Basu D and Abumrad NA: Lipolytic enzymes and free fatty acids at the endothelial interface. Atherosclerosis 329: 1-8, 2021

12. Su YR, Hong YP, Mei FC, Wang CY, Li M, Zhou Y, Zhao KL, Yu J and Wang WX: High-Fat diet aggravates the intestinal barrier injury via TLR4-rip3 pathway in a rat model of severe acute pancreatitis. Mediators Inflamm 2019: 2512687, 2019.

13. Tanaka S, Nemoto Y, Takei Y, Morikawa R, Oshima S, Nagaishi T, Okamoto R, Tsuchiya K, Nakamura T, Stutte $S$ and Watanabe M: High-fat diet-derived free fatty acids impair the intestinal immune system and increase sensitivity to intestinal epithelial damage. Biochem Biophys Res Commun 522: 971-977, 2020.

14. Svitkina TM: Ultrastructure of the actin cytoskeleton. Curr Opin Cell Biol 54: 1-8, 2018.

15. Van Itallie CM, Tietgens AJ, Krystofiak E, Kachar B and Anderson JM: A complex of ZO-1 and the BAR-domain protein TOCA-1 regulates actin assembly at the tight junction. Mol Biol Cell 26: 2769-2787, 2015.

16. Malinova TS and Huveneers S: Sensing of cytoskeletal forces by asymmetric adherens junctions. Trends Cell Biol 28: 328-341, 2018.

17. Yonemura S: Actin filament association at adherens junctions. J Med Invest 64: 14-19, 2017.

18. Shakhov AS, Dugina VB and Alieva IB: Structural features of actin cytoskeleton required for endotheliocyte barrier function. Biochemistry (Mosc) 84: 358-369, 2019.

19. Pollard TD: Actin and actin-binding proteins. Cold Spring Harb Perspect Biol 8: a018226, 2016.

20. Petersen OH, Tepikin AV, Gerasimenko JV, Gerasimenko OV, Sutton R and Criddle DN: Fatty acids, alcohol and fatty acid ethyl esters: Toxic $\mathrm{Ca}^{2+}$ signal generation and pancreatitis. Cell Calcium 45: 634-642, 2009.

21. Bürgin-Maunder CS, Brooks PR and Russell FD: Omega-3 fatty acids modulate Weibel-Palade body degranulation and actin cytoskeleton rearrangement in PMA-stimulated human umbilical vein endothelial cells. Mar Drugs 11: 4435-4450, 2013.

22. Izadi M, Hou W, Qualmann B and Kessels MM: Direct effects of $\mathrm{Ca}^{2+} /$ calmodulin on actin filament formation. Biochem Biophys Res Commun 506: 355-360, 2018.

23. Feldt J, Schicht M, Garreis F, Welss J, Schneider UW and Paulsen F: Structure, regulation and related diseases of the actin-binding protein gelsolin. Expert Rev Mol Med 20: e7, 2019

24. Chan MW, El Sayegh TY, Arora PD, Laschinger CA, Overall CM, Morrison C and McCulloch CA: Regulation of intercellular adhesion strength in fibroblasts. J Biol Chem 279: 41047-41057, 2004.

25. Kim KM, Adyshev DM, Kása A, Zemskov EA, Kolosova IA Csortos C and Verin AD: Putative protein partners for the human CPI-17 protein revealed by bacterial two-hybrid screening. Microvasc Res 88: 19-24, 2013.

26. Livak KJ and Schmittgen TD: Analysis of relative gene expression data using real-time quantitative PCR and the 2(-Delta Delta C(T)) method. Methods 25: 402-408, 2001.

27. Kojima T, Yamaguchi H, Ito T, Kyuno D, Kono T, Konno T and Sawada N: Tight junctions in human pancreatic duct epithelial cells. Tissue Barriers 1: e24894, 2013.

28. Naydenov NG, Baranwal S, Khan S, Feygin A, Gupta P and Ivanov AI: Novel mechanism of cytokine-induced disruption of epithelial barriers: Janus kinase and protein kinase D-dependent downregulation of junction protein expression. Tissue Barriers 1: e25231, 2013.

29. Sakakibara S, Maruo T, Miyata M, Mizutani K and Takai Y: Requirement of the F-actin-binding activity of l-afadin for enhancing the formation of adherens and tight junctions. Genes Cells 23: 185-199, 2018.

30. Lee M and Kang EH: Molecular dynamics study of interactions between polymorphic actin filaments and gelsolin segment-1. Proteins 88: 385-392, 2020

31. Feng S, Wei Q, Hu Q, Huang X, Zhou X, Luo G, Deng M and Lü M: Research progress on the relationship between acute pancreatitis and calcium overload in acinar cells. Dig Dis Sci 64: 25-38, 2019.

32. Nag S, Larsson M, Robinson RC and Burtnick LD: Gelsolin: The tail of a molecular gymnast. Cytoskeleton (Hoboken) 70: 360-384, 2013. 
33. Kang B, Jo S, Baek J, Nakamura F, Hwang W and Lee H: Role of mechanical flow for actin network organization. Acta Biomater 90: 217-224, 2019.

34. Odenwald MA, Choi W, Buckley A, Shashikanth N, Joseph NE, Wang Y, Warren MH, Buschmann MM, Pavlyuk R, Hildebrand J, et al: ZO-1 interactions with F-actin and occludin direct epithelial polarization and single lumen specification in 3D culture. J Cell Sci 130: 243-259, 2017.

35. Troyanovsky RB, Indra I, Chen CS, Hong S and Troyanovsky SM: Cadherin controls nectin recruitment into adherens junctions by remodeling the actin cytoskeleton. J Cell Sci 128: 140-149, 2015.

36. McRae M, LaFratta LM, Nguyen BM, Paris JJ, Hauser KF and Conway DE: Characterization of cell-cell junction changes associated with the formation of a strong endothelial barrier. Tissue Barriers 6: e1405774, 2018
37. Sluysmans S, Vasileva E, Spadaro D, Shah J, Rouaud F and Citi S: The role of apical cell-cell junctions and associated cytoskeleton in mechanotransduction. Biol Cell 109: 139-161, 2017.

38. Becker PM, Kazi AA, Wadgaonkar R, Pearse DB, Kwiatkowski D and Garcia JG: Pulmonary vascular permeability and ischemic injury in gelsolin-deficient mice. Am J Respir Cell Mol Biol 28: 478-484, 2003.

39. Bogatcheva NV and Verin AD: The role of cytoskeleton in the regulation of vascular endothelial barrier function. Microvasc Res 76: 202-207, 2008.

cc) (i) () $($ This work is licensed under a Creative Commons c) Attribution-NonCommercial-NoDerivatives 4.0 International (CC BY-NC-ND 4.0) License. 